ANNALES

POLONICI MATHEMATICI

$96.1(2009)$

\title{
Cousin-I spaces and domains of holomorphy
}

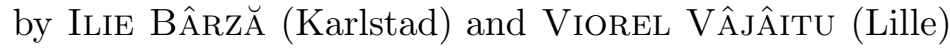

\begin{abstract}
We prove that a Cousin-I open set $D$ of an irreducible projective surface $X$ is locally Stein at every boundary point which lies in $X_{\text {reg. }}$. In particular, Cousin-I proper open sets of $\mathbb{P}^{2}$ are Stein. We also study $K$-envelopes of holomorphy of $K$-complete spaces.
\end{abstract}

1. Introduction. Let $X=\left(X, \mathcal{O}_{X}\right)$ be a complex space. We denote by $\mathcal{M}_{X}$ the sheaf of germs of meromorphic functions on $X$. Following Grauert and Remmert ([9, p. 141]), we say that $X$ has the Cousin-I property or that $X$ is a Cousin-I space if the first (additive) Cousin problem is universally solvable on $X$.

This means that, for every Cousin data $\left\{\left(U_{i}, m_{i}\right)\right\}_{i}$ on $X$ (i.e. $\left\{U_{i}\right\}_{i}$ is an open covering of $X$ and $m_{i}$ is a meromorphic function on $U_{i}$ such that on the (non-empty) intersection $U_{i} \cap U_{j}$ the difference $m_{i}-m_{j}$ is holomorphic) there is a meromorphic function $m$ on $X$ such that $\left.m\right|_{U_{i}}-m_{i}$ is holomorphic on $U_{i}$. Reformulated in cohomological language, this reads: the natural map $H^{0}\left(X, \mathcal{M}_{X}\right) \rightarrow H^{0}\left(X, \mathcal{M}_{X} / \mathcal{O}_{X}\right)$ is surjective, or equivalently, the morphism $H^{1}\left(X, \mathcal{O}_{X}\right) \rightarrow H^{1}\left(X, \mathcal{M}_{X}\right)$ is injective.

Consequently, a reasonable sufficient condition for $X$ to be a Cousin-I space is the vanishing of $H^{1}\left(X, \mathcal{O}_{X}\right)$. However, there are smooth compact $K 3$ surfaces $M$ with $H^{1}\left(M, \mathcal{O}_{M}\right) \neq 0$ and no complex curve on $M$, so that $M$ is trivially a Cousin-I space. (This is because on a complex manifold $Z$ the existence of non-trivial first Cousin data implies that there are complex hypersurfaces on $Z$.)

Although being a Cousin-I space is an intrinsic property, the notion is extrinsically studied, usually for open sets in a larger space having "many holomorphic functions". For instance, it was shown in [2] that a Cousin-I open set in a Stein surface is itself Stein. This extends a well-known theorem due to Cartan, Behnke and Stein ([4], [5], [3]). We prove:

2000 Mathematics Subject Classification: 32D05, 32D10, 32D26, 32E10.

Key words and phrases: Cousin-I space, domain of holomorphy, $K$-complete space, Stein space. 
Theorem 1. Let $X$ be an irreducible projective surface and $D \subset X a$ Cousin-I open set. Then $D$ is locally Stein at every point $x_{0} \in \partial D \cap X_{\text {reg }}$. In particular, Cousin-I proper open sets of $\mathbb{P}^{2}$ are Stein.

In this circle of ideas we also prove (see $\S 4$ for definitions):

Proposition 1. Let $X$ be a $K$-complete, connected, normal complex surface. If $X$ is a Cousin-I space, then $X$ coincides with its $K$-envelope of holomorphy.

It turns out that when dealing with these questions it is necessary to reconsider the notion of domain of holomorphy in complex spaces. This is done in $\S 2$.

2. Preliminaries. Throughout this paper complex spaces are not necessarily reduced. Curves, surfaces, etc. will be complex spaces of appropriate pure dimensions.

Before giving the notion of domain of holomorphy we employ, let us give an example of an irreducible Stein curve $X$ and a non-empty open set $D \subset X$ for which there are two non-empty open sets $U_{1}, U_{2}$ such that $U_{2}$ is connected, $U_{1} \subset D \cap U_{2} \neq U_{2}$, and for every holomorphic function $f$ on $D$ there is a holomorphic function $f_{2}$ on $U_{2}$ such that $f=f_{2}$ on $U_{1}$.

Let $X$ be the node $\left\{(z, w) \in \mathbb{C}^{2} ; w^{2}=z^{2}+z^{3}\right\}$ and $\pi: \mathbb{C} \rightarrow X$ the normalization map given by

$$
\pi(t)=\left(t^{2}-1, t\left(t^{2}-1\right)\right), \quad t \in \mathbb{C} .
$$

Clearly, $X$ is an irreducible Stein curve which is not locally irreducible at its (only) singular point $x_{0}=(0,0), \pi^{-1}\left(x_{0}\right)=\{-1,1\}$, and $\pi$ induces a biholomorphism between $\mathbb{C} \backslash\{-1,1\}$ and $X \backslash\left\{x_{0}\right\}$.

We remark that if $S$ and $T$ are disjoint subsets of $\mathbb{C}$ such that $S \ni-1$ and $T \ni 1$, then $\pi(S) \cap \pi(T)=\left\{x_{0}\right\}$. Now take $S$ and $T$ open and connected; let $S^{\prime}$ and $T^{\prime}$ be non-empty connected open subsets of $\mathbb{C}$ such that $S^{\prime} \subset S$, $-1 \notin S^{\prime}, T^{\prime} \subset T, T^{\prime} \neq T$ and $1 \in T^{\prime}$. Put $D=\pi\left(S \cup T^{\prime}\right), U_{1}=\pi\left(S^{\prime}\right)$ and $U_{2}=\pi(S \cup T)$. Clearly, $D$ is a Stein open set in $X, U_{1}$ and $U_{2}$ are non-empty open sets, $U_{2}$ is connected and $U_{1} \subset U_{2} \cap D \neq U_{2}$. However, for each $f \in \mathcal{O}(D)$ there exists $f_{2} \in \mathcal{O}\left(U_{2}\right)$ with $f=f_{2}$ on $U_{1}$. (Notice that $\pi(S)$ and $\pi\left(T^{\prime}\right)$ are the irreducible components of $D$.)

This shows that the ordinary definition of domain of holomorphy from the smooth case does not carry over ad litteram to complex spaces. The appropriate one is:

Definition 1. Let $X$ be a complex space of pure dimension $n$. An open set $D$ in $X$ is a domain of holomorphy if there do not exist non-empty open sets $U_{1}$ and $\widehat{U}_{2}$ of $X$ and an irreducible component $Y$ of $X$ such that: 
(i) the analytic set $U_{2}:=\widehat{U}_{2} \cap Y$ is irreducible,

(ii) $U_{1} \subset U_{2} \cap D$ and $U_{2} \not \subset D$,

(iii) for every $f \in \mathcal{O}(D)$ there exists $f_{2} \in \mathcal{O}\left(U_{2}\right)$ with $f=f_{2}$ on $U_{1}$.

The key result to deal with this definition is the construction of irreducible principal Stein surfaces in Stein spaces.

Theorem 2. Let $X$ be a Stein space of pure dimension n. Let $Y \subset X$ be a locally analytic set which is Stein, irreducible and of dimension $n$. Let also $\Lambda$ and $\Lambda^{\prime}$ be two disjoint discrete subsets in $X$. Then there exists a holomorphic function $f \in \mathcal{O}(X)$ with the following properties:

(a) The set $Z_{f}:=\{f=0\}$ contains $\Lambda$ and is disjoint from $\Lambda^{\prime}$.

(b) The analytic set $Y \cap Z_{f}$ is irreducible and $f$ has multiplicity one on it.

The proof is similar to the one in [16] so we confine ourselves to indicating the necessary changes. For instance, on p. 202 the "almost-proper" holomorphic map $\pi$ should now be considered from $Y$ into $\mathbb{C}^{n}$. Also, there we did not have a condition concerning $\Lambda^{\prime}$. However, this can be easily satisfied as we reasoned using Baire's theorem and because $f$ is of the form $f=F_{g}:=\sum_{i=1}^{m} g_{i} h_{i}$, with fixed $h_{1}, \ldots, h_{m} \in \mathcal{O}(X)$ such that $\Lambda=\left\{h_{1}=\right.$ $\left.\cdots=h_{m}=0\right\}$ and $g=\left(g_{1}, \ldots, g_{m}\right)$ running over a $G_{\delta}$ set. The simple observation that $\left\{g \in \mathcal{O}(X)^{m} ; \exists a \in \Lambda^{\prime}, F_{g}(a) \neq 0\right\}$ is open and dense in $\mathcal{O}(X)^{m}$ concludes the proof of the theorem.

Proposition 2. Let $X$ be a Stein space of pure dimension $n$. Let $\Omega$ be a Cousin-I open set in $X$. Then $\Omega$ is a domain of holomorphy provided that the following condition $(\star)$ holds:

$(\star)$ For each holomorphic function $f \in \mathcal{O}(X)$ which is non-constant on every irreducible component of $X$ of positive dimension, $\Omega \cap f^{-1}(0)$ is a domain of holomorphy in $f^{-1}(0)$.

Proof. For an arbitrary complex space $X$, let $S_{d}=\left\{x \in X\right.$; prof $\mathcal{O}_{X, x} \leq$ $d\}$. Then $S_{d}$ is an analytic set in $X$ of dimension $\leq d$. It is easily seen that there is a discrete set $\Lambda=\left\{x_{i}\right\}_{i \in I}$ of points in $X$ (possibly infinite) such that, for each $d=0,1, \ldots$, every $d$-dimensional irreducible component of $S_{d}$ contains a point of $\Lambda$. Now, if $f$ is a holomorphic function on $X$ and $f\left(x_{i}\right) \neq 0$ for all $i$, then by [14], for every $x \in X$, the germ $f_{x}$ is not a zero divisor in $\mathcal{O}_{X, x}$ so that the multiplication morphism induced by $f$ from $\mathcal{O}_{X}$ into itself is injective.

Now we come to the proof of the proposition. Assume, in order to reach a contradiction, that $\Omega$ is not a domain of holomorphy in $X$; let $U_{1}$ and $U_{2}$ be according to the definition. We can assume that $U_{2}$ is Stein. Let $\widetilde{U}_{1}$ be the union of the irreducible components of $U_{2} \cap \Omega$ meeting $U_{1}$. Using the 
normalization map we see that there is a boundary point $a$ of $\Omega$ that is also a boundary point of $\widetilde{U}_{1}$. (This a might belong to the set $\Lambda$ above.) Fix a point $b \in U_{1} \backslash \Lambda$. By Theorem 2, there exists $f \in \mathcal{O}(X)$ with the following properties:

(1) The zero set $Z$ of $f$ contains the points $a$ and $b$ and for each $x \in$ $\Lambda \backslash\{a\}, f(x) \neq 0$.

(2) The analytic set $U_{2} \cap Z$ is irreducible.

Let $Z$ be endowed with the full fiber of $f$ over $0 \in \mathbb{C}$ and $\Omega^{\prime}:=\Omega \cap Z$. We claim that $\Omega^{\prime}$ is not a domain of holomorphy in $Z$. To show this, first observe that $U_{1}^{\prime}:=U_{1} \cap Z$ and $U_{2}^{\prime}:=U_{2} \cap Z$ are as in the definition. Now let $h \in \mathcal{O}\left(\Omega^{\prime}\right)$. From [2, p. 321], since $f_{x}$ is not a zero divisor of $\mathcal{O}_{X, x}$, for every $x \in \Omega$, there exists $H \in \mathcal{O}(\Omega)$ which extends $h$. But, in view of our assumption, there exists $H_{2} \in \mathcal{O}\left(U_{2}\right)$ which agrees with $H$ on $U_{1}$. The claim follows and so the proof of the proposition is complete.

REMARK. This corollary generalizes Cartan's well-known result for Cousin-I open sets in $\mathbb{C}^{2}$.

3. Proof of Theorem 1. The proof is divided into several steps and is completed in the last fifth step.

STEP 1. Let $X=\left(X, \mathcal{O}_{X}\right)$ be a complex space. We say that $X$ is $c o$ homologically 1-convex if, and only if, for any coherent analytic sheaf $\mathcal{F}$ on $X$, the cohomology groups $H^{j}(X, \mathcal{F}), j=1,2, \ldots$, are finite-dimensional complex vector spaces. For instance, a complex curve is cohomologically 1-convex if, and only if, it has finitely many one-dimensional compact irreducible components. For more details, see [12].

Also, if $Y=\left(Y, \mathcal{O}_{Y}\right)$ is another complex space, then we say that $Y$ is an infinitesimal extension of $X$ if they have the same underlying topological space and $X$ becomes a closed subspace of $Y$, that is, there is a coherent sheaf of ideals $\mathcal{I} \subseteq \mathcal{O}_{Y}$ with $\operatorname{Supp}\left(\mathcal{O}_{Y} / \mathcal{I}\right)=X$ and $\mathcal{O}_{X} \simeq \mathcal{O}_{Y} / \mathcal{I}$. From [17] we get:

LEMMA 1. Let $X$ and $Y$ be a complex spaces such that $Y$ is an infinitesimal extension of $X$. Then $X$ and $Y$ are simultaneously cohomologically 1-convex.

SteP 2. Let $X$ be a projective space and $L$ an ample line bundle over $X$. Let $\sigma \in \Gamma(X, L), \sigma \neq 0$. Put $X_{0}:=X \backslash\{\sigma=0\}$. Then the set

$$
\left\{\left.\left(\tau / \sigma^{m}\right)\right|_{X_{0}} ; \tau \in \Gamma\left(X, L^{m}\right), m \in \mathbb{N}\right\}
$$

is dense in $\mathcal{O}\left(X_{0}\right)$ with respect to the canonical topology of uniform convergence on compact subsets of $X_{0}$. 
Indeed, it is no loss of generality to assume that $L$ is the restriction of $\mathcal{O}(1)$ to $X$ for some holomorphic embedding $X \hookrightarrow \mathbb{P}^{N}$ and $\sigma$ comes from a hyperplane section. Now, because $X_{0}$ is realized as a closed analytic subspace of $\mathbb{C}^{N}=\mathbb{P}^{N} \backslash\{\sigma=0\}$, any holomorphic function on $X_{0}$ is the restriction of a holomorphic function on $\mathbb{C}^{N}$ which can be approximated by polynomials. The proof of Step 1 concludes easily.

SteP 3. Let $X$ be a Cousin-I complex space and $L$ a holomorphic line bundle over $X$. Assume that there is a section $\sigma \in \Gamma(X, L)$ such that the multiplication by $\sigma$ induces an injective $\mathcal{O}_{X}$-morphism $\mathcal{O}_{X} \rightarrow \mathcal{L}$, where $\mathcal{L}$ denotes the sheaf of germs of holomorphic sections in $L$. Then the canonical restriction map

$$
H^{0}(X, \mathcal{L}) \rightarrow H^{0}\left(X, \mathcal{L} / \sigma \mathcal{O}_{X}\right)
$$

is surjective. We notice that the above injectivity results if $X$ is irreducible and $\sigma \neq 0$. (For $s \in H^{0}\left(X, \mathcal{L} / \sigma \mathcal{O}_{X}\right)$, select a locally finite Stein open covering $\left\{V_{i}\right\}_{i}$ of $X$ and then extensions $s_{i} \in H^{0}\left(V_{i}, \mathcal{L}\right)$ of $\left.s\right|_{V_{i}}$. It follows readily that $\left\{\left(V_{i}, s_{i} / \sigma\right)\right\}_{i}$ defines an additive Cousin data on $X$. Let $m \in \mathcal{M}(X)$ be a solution. Then $m \sigma$ is the desired extension.)

STEP 4. For a complex space $X$ and a holomorphic function $f$ on $X$, let $Z_{f}$ be the zero set of $f$, i.e. $Z_{f}=\{x \in X ; f(x)=0\}$. From [16] we recall the following result.

Lemma 2. Let $X$ be an irreducible Stein space and $D$ an irreducible open set in $X$ which is Stein and Runge in $X$. Then, for any $x_{1}, x_{2} \in D$, there exists a holomorphic function $f$ on $X$ such that $x_{1}$ and $x_{2}$ are contained in the same irreducible component of $Z_{f} \cap D$.

Furthermore, if $U_{1}$ is an open neighborhood of $x_{1}$, then there is a compact set $K$ in $D$ and $\varepsilon>0$ such that, for any holomorphic function $h$ on $X$ with

$$
h\left(x_{2}\right)=0 \quad \text { and } \max _{x \in K}|h(x)-g(x)| \leq \varepsilon,
$$

there is an irreducible component $\Sigma$ of $Z_{h}$ passing through $x_{2}$ such that $\Sigma \cap U_{1}$ is non-empty and $\Sigma \cap D$ is irreducible.

SteP 5. Now we conclude the proof of Theorem 1. Let $X \hookrightarrow \mathbb{P}^{N}$ be a holomorphic embedding such that there are finitely many global holomorphic sections $\sigma_{j} \in \Gamma\left(\mathbb{P}^{N}, \mathcal{O}(1)\right)$ such that the singular part $X_{\text {sing }}$ of $X$ is $X \cap\left\{\sigma_{1}=\cdots=\sigma_{m}=0\right\}$.

Let $\sigma$ be one of the sections $\sigma_{1}, \ldots, \sigma_{m}$. We show that $D_{0}:=D \backslash\{\sigma=0\}$ is Stein (in the Stein smooth connected surface $X_{0}:=X \backslash\{\sigma=0\}$ ).

Assume, in order to reach a contradiction, that $D_{0}$ is not Stein. Therefore, due to the positive solution of the Levi problem in Stein manifolds, there is a point $a \in \partial D \cap X_{0}$ such that, for every Stein open neighborhood $W$ of $a$ in $X_{0}, W \cap D$ is not Stein. Hence there are non-empty connected 
open sets $U_{1}$ and $U_{2}$ in $W$ such that $U_{1} \subset D \cap U_{2} \neq U_{2}, U_{2}$ is Stein and Runge in $X_{0}$, and for every holomorphic function $F$ on $W \cap D$, there is a holomorphic function $F_{2}$ on $U_{2}$ such that $F=F_{2}$ on $U_{1}$. Clearly, we may assume that $U_{1}$ is a connected component of $U_{2} \cap D$.

From Steps 2 and 4 (and replacing $L$ by a suitable tensor power of it, if necessary), there is a holomorphic section $\tau \in \Gamma(X, L), \tau \neq 0$, and an irreducible component $\Sigma$ of the compact complex curve $\{\tau=0\}$ such that $\Sigma \ni a, \Sigma \cap U_{2}$ is irreducible and $\Sigma \cap U_{1} \neq \emptyset$. This implies easily that the boundary of $U_{1} \cap \Sigma$ computed with respect to $U_{2} \cap \Sigma$ is non-empty. Therefore, there is a boundary point $a_{\sharp}$ of $D$ in $U_{2}$ that is also an accumulation point of $U_{1} \cap \Sigma$.

Let $X^{\prime}:=\{x \in X ; \tau(x)=0\}$. This is a compact curve and $D^{\prime}:=D \cap X^{\prime}$ is cohomologically 1-convex. Let $\mathcal{I}$ be the ideal sheaf of $X^{\prime}$ in $X, L^{\prime}$ the restriction of $L$ to $X^{\prime}$ (which is again a holomorphic line bundle), $\mathcal{L}^{\prime}$ the sheaf of germs of its holomorphic sections (which is a coherent $\mathcal{O}_{X^{\prime}}$-module) and $\mathcal{S}$ the sheaf of germs of holomorphic sections of $L$ that vanish on $X^{\prime}$.

Now, because $X^{\prime}$ is compact, there is a positive integer $m$ such that $\mathcal{I}^{m} \mathcal{S} \subseteq \sigma \mathcal{O}_{X}$. This implies that $\mathcal{S} / \sigma \mathcal{O}_{X}$ is $\mathcal{O}_{X} / \mathcal{I}^{m}$-coherent. Thus, by Step 1, the cohomology group $H^{1}\left(D^{\prime}, \mathcal{S} / \sigma \mathcal{O}_{X}\right)$ has finite dimension.

To reach the desired contradiction, let $\left\{x_{\nu}\right\}_{\nu}$ be a sequence of points in $D^{\prime}$ converging to $a_{\sharp}$. Since $D^{\prime}$ is holomorphically convex, there is a holomorphic function $f$ on $D^{\prime}$ such that $\left\{f\left(x_{\nu}\right)\right\}_{\nu}$ is unbounded in $\mathbb{C}$. Let also $\theta \in \Gamma(X, L)$ with $\theta\left(a_{\sharp}\right) \neq 0$ and let $\theta^{\prime} \in \Gamma\left(X^{\prime}, L^{\prime}\right)$ be canonically induced by $\theta$. On the other hand, the short exact sequence

$$
0 \rightarrow \mathcal{S} / \sigma \mathcal{O}_{X} \rightarrow \mathcal{L} / \sigma \mathcal{O}_{X} \rightarrow \mathcal{L}^{\prime} \rightarrow 0
$$

induces a long exact cohomology sequence from which we retain the exact portion

$$
H^{0}\left(D^{\prime}, \mathcal{L} / \sigma \mathcal{O}_{X}\right) \rightarrow H^{0}\left(D^{\prime}, \mathcal{L}^{\prime}\right) \rightarrow H^{1}\left(D^{\prime}, \mathcal{S} / \sigma \mathcal{O}_{X}\right) .
$$

But, as $H^{1}\left(D^{\prime}, \mathcal{S} / \sigma \mathcal{O}_{X}\right)$ has finite dimension, there is a non-constant holomorphic polynomial $P$ in one complex variable such that $P(f) \theta^{\prime} \in H^{0}\left(D^{\prime}, \mathcal{L}^{\prime}\right)$ comes from a holomorphic section $\widehat{\theta} \in H^{0}(D, \mathcal{L})$. Then $F:=\widehat{\theta} / \sigma$ is holomorphic on $D_{0}$ and is unbounded near $a_{\sharp}$, so that no holomorphic "extension" $F_{2}$ exists. The conclusion of the theorem follows. (The additional statement now follows easily thanks to the positive solution of the Levi problem in $\mathbb{P}^{k}$.)

4. Proof of Proposition 1. The material presented so far motivates the question of characterizing those $K$-complete complex spaces $X$ that are Cousin-I spaces. Below we give a description of $X$ in terms of certain envelopes of holomorphy; as a byproduct, this will give a proof of Proposition 1 . 
First we recall a few concepts. Let $X$ be a complex space. We say that $X$ is $K$-complete if, for any point $x_{0}$ of $X$, there exists a holomorphic mapping $F: X \rightarrow \mathbb{C}^{m}, m=m\left(x_{0}\right)$, such that $x_{0}$ is isolated in the fiber $F^{-1}\left(F\left(x_{0}\right)\right)$ and $F$ is nowhere degenerate $\left({ }^{1}\right)$ in a neighborhood of $x_{0}$.

Let $X$ be $K$-complete. By a $K$-envelope of holomorphy of $X$ we mean a pair $(\alpha, \widetilde{X})$ with the following two properties:

(i) $\tilde{X}$ is a complex space and $\alpha: X \rightarrow \widetilde{X}$ is a nowhere degenerate holomorphic map such that the natural homomorphism ${ }^{\star} \alpha: \mathcal{O}(\widetilde{X}) \rightarrow$ $\mathcal{O}(X)$ induced by $\alpha$ is a ring isomorphism.

(ii) For each $K$-complete complex space $Y$ and for every nowhere degenerate holomorphic map $\beta: X \rightarrow Y$ such that ${ }^{\star} \beta: \mathcal{O}(Y) \rightarrow \mathcal{O}(X)$ is a ring isomorphism ( ${ }^{\star} \beta$ is induced by $\beta$ ), there is a nowhere degenerate holomorphic mapping $h: Y \rightarrow \widetilde{X}$ which makes the following diagram commutative:

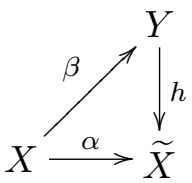

It is proved in [11] that the $K$-envelope of holomorphy $(\alpha, \widetilde{X})$ exists and it is uniquely determined (up to analytic isomorphism) for $X$ connected, normal and $K$-complete. Also, $\widetilde{X}$ is $K$-complete, normal and connected.

In order to introduce the second type of envelope recall that a branched Riemann domain over $\mathbb{C}^{n}$ is a pair $(X, \pi)$ where $X$ is a reduced complex space and $\pi: X \rightarrow \mathbb{C}^{n}$ a nowhere degenerate holomorphic map. To such a branched Riemann domain $(X, \pi)$ we associate the set $\mathcal{D}$ of all Riemann domains $(Y, \sigma)$ over $\mathbb{C}^{n}$ such that there exists a commutative diagram of holomorphic maps

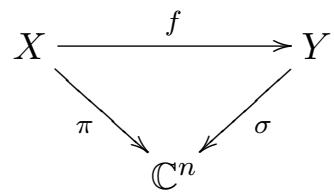

for which the naturally induced map ${ }^{\star} f: \mathcal{O}(Y) \rightarrow \mathcal{O}(X)$ is bijective. On $\mathcal{D}$ we define a natural ordering " $\prec$ " by saying that $\left(Y_{1}, \sigma_{1}\right) \prec\left(Y_{2}, \sigma_{2}\right)$ if there exists a holomorphic map $h: Y_{1} \rightarrow Y_{2}$ with $\sigma_{2} \circ h=\sigma_{1}$ and ${ }^{\star} h: \mathcal{O}\left(Y_{2}\right) \rightarrow$ $\mathcal{O}\left(Y_{1}\right)$ is bijective. It is shown in [11] that $\mathcal{D}$ has a maximal element $(\widehat{X}, \widehat{\pi})$

$\left({ }^{1}\right)$ A mapping $u: S \rightarrow T$ between connected topological spaces is said to be nowhere degenerate if each fiber of $u$ is a discrete set in $S$ and the image $u(S)$ contains an open subset of $T$. 
(unique up to analytic isomorphism), which we call the Thullen-Cartan envelope of $(X, \pi)$.

The relation between $\widetilde{X}$ and $\widehat{X}$ is clarified in [11]. Let $X$ be a connected, normal, $K$-complete complex space. Let $(\alpha, \widetilde{X})$ be the $K$-envelope of holomorphy of $X$ and let $\widetilde{\pi}: \widetilde{X} \rightarrow \mathbb{C}^{n}$ be the holomorphic extension of $\pi: X \rightarrow \mathbb{C}^{n}$. Then $\widehat{X}$ might be viewed as the complement in $\widetilde{X}$ of the degeneracy set of $\tilde{\pi}$.

Proposition 3 ([11]). Let $X$ be a $K$-complete, normal, connected complex space. Then $X$ coincides with its $K$-envelope of holomorphy $\widetilde{X}$ if and only if, for any realization $(X, \pi)$ of $X$ as a Riemann domain over $\mathbb{C}^{n}$, $(X, \pi)$ coincides with $(\widehat{X}, \widehat{\pi})$.

Now we give:

Proposition 4. Let $X$ be a connected, normal $K$-complete space. If $X$ is a Cousin-I space and for each non-constant holomorphic function $f$ on $X$, $\{f=0\}$ is Stein, then $X$ coincides with its $K$-envelope of holomorphy.

Proof. It suffices to establish the following fact. Let $Y$ be a $K$-complete holomorphic extension of $X$, that is, $Y$ is connected, normal, $K$-complete, and there is a nowhere degenerate holomorphic map $\beta: X \rightarrow Y$ such that ${ }^{\star} \beta: \mathcal{O}(Y) \rightarrow \mathcal{O}(X)$ is a ring isomorphism. Under the hypothesis of Proposition 4, we show that $\beta$ is biholomorphic. We do this in two steps.

STEP 1. We show that $\mathcal{O}(X)$ separates the points of $X$, so that $\beta$ is injective; as $X$ and $Y$ are normal, $\beta$ is also an open map, so that $X$ may be regarded as an open subset of $Y$.

To check this step, let $x \neq y$ be two points of $X$. Let $f$ be a nonconstant holomorphic function on $X$. If $f(x) \neq f(y)$, then we are done. If $f(x)=f(y)=: c$, then the hypothesis implies that the restriction map $\mathcal{O}(X) \rightarrow \mathcal{O}(Y)$ is surjective, where $Y=\{f=c\}$. Since $Y$ is Stein, the assertion follows.

STEP 2. The map $\beta$ is surjective. In order to prove this we shall apply the following proposition which results mutatis mutandis from [16] (see the proof of Theorem 2 there).

Proposition 5. Let $Z$ be an irreducible $K$-complete complex space and $\Gamma$ a discrete subset of $Z$. Then there exists a holomorphic function $f$ on $Z$ for which there is a (unique) irreducible component of $\{f=0\}$ containing $\Gamma$.

Now, returning to the proof of Step 2, assume, in order to reach a contradiction, that there exists $x_{0} \in \partial X$. Let $\widehat{f}$ be a non-constant holomorphic function on $\widehat{X}$ such that there is an irreducible component $\Sigma$ of $\{\widehat{f}=0\}$ 
containing $x_{0}$ and intersecting $X$. Now the boundary of $\Sigma \cap X$ (computed in $\Sigma$ ) cannot be empty. (Otherwise, $\Sigma \cap X$ is closed and open in $\Sigma$, thus $\Sigma \cap X$ is a union of connected components of $\Sigma$, thus $\Sigma \cap X$ equals $\Sigma$ because $\Sigma$ is irreducible, a fortiori connected. This gives $x_{0} \in X$, which is not the case.) On the other hand, one verifies easily that $\partial_{\Sigma}(\Sigma \cap X) \subset \Sigma \cap \partial X$. Thus there is a boundary point of $X$ which is an accumulation point of $\Sigma \cap X$, which means that there is a sequence of points $\left\{x_{\nu}\right\}_{\nu}$ in $X \cap\{\widehat{f}=0\}$ converging to a point of $\partial X$. Let $g$ be a holomorphic function on $X$ such that $\left\{g\left(x_{\nu}\right)\right\}_{\nu}$ is unbounded. Considering its holomorphic extension $\widehat{g}$ to $\widehat{X}$ we arrive easily at a contradiction. This implies the surjectivity of $\beta$, whence Step 2.

\section{References}

[1] M. Abe and Y. Abe, Domains over a K-complete manifold, Mem. Fac. Sci. Kyushu Univ. Ser. A 38 (1984), 133-140.

[2] E. Ballico, On Cousin-I complex spaces, Enseign. Math. (2) 27 (1981), 321-325.

[3] H. Behnke und K. Stein, Analytische Funktionen mehrerer Veränderlichen zu vorgegebenen Null- und Polstellenflächen, Jahresber. Deutsch. Math.-Verein. 47 (1937), 177-192.

[4] H. Cartan, Sur les problèmes de Poincaré et de Cousin pour les fonctions de plusieurs variables complexes, C. R. Acad. Sci. Paris Sér. A-B 199 (1934), 1284-1287.

[5] —, Sur le premier problème de Cousin, ibid. 207 (1938), 558-560.

[6] J.-P. Demailly, Courants positifs extrêmaux et conjecture de Hodge, Invent. Math. 69 (1982), 347-374.

[7] R. Fujita, Domaines sans point critique intérieur sur l'espace projectif complexe, J. Math. Soc. Japan 15 (1963), 443-473.

[8] H. Grauert, Charakterisierung der holomorph vollständigen komplexen Räume, Math. Ann. 129 (1955), 233-259.

[9] H. Grauert und R. Remmert, Theorie der Steinschen Räume, Grundlehren Math. Wiss. 227, Springer, 1977.

[10] J. Kajiwara, Some extensions of Cartan-Behnke-Stein's theorem, Publ. Res. Inst. Math. Sci. Ser. A 2 (1966), 133-156.

[11] H. Kerner, Holomorphiehüllen zu K-vollständigen komplexen Räumen, Math. Ann. 138 (1959), 316-328.

[12] R. Narasimhan, The Levi problem for complex spaces II, ibid. 142 (1960/1961), 355-365.

[13] G. Scheja, Verzweigte Holomorphiehüllen, S.-B. Bayer. Akad. Wiss. Math.-Natur. Kl. 1958, 9-18.

[14] Y.-T. Siu and G. Trautmann, Gap-sheaves and Extension of Coherent Analytic Subsheaves, Lecture Notes in Math. 172, Springer, 1971.

[15] T. Ueda, Domains of holomorphy in Segre cones, Publ. Res. Inst. Math. Sci. 22 (1986), 561-569.

[16] V. Vâjâitu, Holomorphic q-hulls in top degrees, Manuscripta Math. 91 (1996), 195210 . 
[17] V. Vâjâitu, Holomorphic convexity of complex spaces with 1-convex hypersections, Forum Math. 17 (2005), 315-323.

Department of Mathematics Karlstad University

SE-651 88 Karlstad, Sweden

E-mail: Ilie.Barza@kau.se
Laboratoire Paul Painlevé Université des Sciences et Technologies de Lille 1

Bât. M2

F-59655 Villeneuve d'Ascq Cedex, France E-mail: Viorel.Vajaitu@math.univ-lille1.fr

Received 21.10.2008

and in final form 3.2.2009 